\title{
Subject Index Vol. 21, 1994
}

Supplement issue No. 1 has its own Subject Index

Angiography, cerebral 243 Anticon|vulsant prophylaxis 165 Ascites 254 Astrocytomas 23 Atlantooccipital dislocation 55, 105

Biodegradation 71

Blood-brain barrier disruption 16

Brain abscess 45

- injury 117

-, neoplasms 232

- tumor 165, 195

Cardiac standstill 124 Carotid occlusion 243 Cerebral dissecting aneurysm 243

- neoplasms 23 Cerebrospinal fluid 66

- - leaks 227

- protein 254

- - shunt(s) 6,71,189 Cervical spine 260 Chiari I malformation 31 Childhood hydrocephalus 59 Children 124 Complications 174 Computed tomography 50, 221, 232 Computer modeling 6 Cranioplasty 77 Craniosynostosis 77

Dextromethorphan 117 Disseminated CNS germinoma 16 Dorsal rhizotomy 237 Down’s syndrome 55 Duraplasty 227

Emergency department 221 Excitotoxicity 117 Extradural hematoma, conservative management 50

False aneurysm 45

Gangliogliomas 23 Gastric perforation 192 
Glioblastomas 23 Gram-negative infection 189

Head injury 50 - -, children 117 Healthcare 36 Hydrocephalus 6,192,227 -, survival analysis 66 Hypsarrhythmia 184

Infantile spasms 184

Intracerebral hematoma, drainage 201

Ischemia 117

-, cerebral 243

Isolated ventricle 59

Magnetic resonance imaging 195, 221, 232,

260 Malignant rhabdoid tumor 232 Meningioma, cystic infantile, malignant

195 Meningitis, aseptic 227 -, medical management 189 Micro vascular neurosurgery 124 Motor vehicle accident 221 Motor-pedestrian accident 221 Moya Moya disease 243 Multidisciplinary clinic 36 Multiple trauma 105 Myelomeningocele 36

Necrobiosis lipoidica 113 Neisseria sicca 189 Neonate 55

Neoplasms, pediatric 23 Neuroprotection 117

Optic glioma 254 Orbital stabs 45 Osteoblastoma 260 Osteoplastic laminotomy 237

Pediatric intensive care unit 221 Penetrating trauma 45 Platinum-based chemotherapy 16 Porencephalic cyst 184 
Posterior fossa tumors 227 Postoperative complications 227

- epilepsy 165

Prognosis 105

Pseudorheumatoid nodule 113

Rabbits 77

Radionuclide shuntogram 71 Replacement laminoplasty 237 Rheumatoid nodule 113

Scoliosis 31 Seizures 23 Shunt 59

- malfunction 71

Silicone 71

Siphon control device 66 Skull 77

- clamp 174

- fractures, depressed 174 Subcutaneous granuloma annulare 113

- palisading granuloma 113 Subdural hematoma 117,201 Subfrontal craniotomy 165 Suboccipital craniectomy 227 Sudden decompression 201 Surgical treatment 184 Suture 77 Syringomyelia 31

Transoral approach 260 Trauma 105

Ventricular catheter placement 59 Ventriculoatrial shunt 254 Ventriculoperitoneal shunt 192, 254 Ventriculopleural shunt 66 Vertebrobasilar giant aneurysms 124

West syndrome 184 